\title{
Serum Amyloid A Protein as a Potential Biomarker Useful in Monitoring the Course of COVID-19 : A Retrospectively Studied
}

\section{Ying Zhang}

Taizhou Hospital, Wenzhou Medical University, Linhai, 317000, China

\section{Donglian Wang}

Taizhou Hospital, Wenzhou Medical University, Linhai, 317000, China

Minjie Lin

Taizhou Hospital, Wenzhou Medical University, Linhai, 317000, China

\section{Tong Sun}

Taizhou Hospital, Wenzhou Medical University, Linhai, 317000, China Jiaxi Chen

Taizhou Hospital, Wenzhou Medical University, Linhai, 317000, China

Jiaqin $\mathrm{Xu}$

Taizhou Hospital, Wenzhou Medical University, Linhai, 317000, China

Hongguo Zhu

Taizhou Hospital, Wenzhou Medical University, Linhai, 317000, China

\section{Guangjun Zhu}

Taizhou Hospital, Wenzhou Medical University, Linhai, 317000, China

\section{Ruyue Lu}

Taizhou Hospital, Wenzhou Medical University, Linhai, 317000, China

\section{Luxiao Hong}

Taizhou Hospital, Wenzhou Medical University, Linhai, 317000, China

\section{Bo Shen}

Taizhou Hospital, Wenzhou Medical University, Linhai, 317000, China

Xiaomai Wu ( $\nabla$ wuxm@enzemed.com )

Taizhou Hospital, Wenzhou Medical University, Linhai, 317000, China

\section{Yufen Zheng ( $\square$ zhengyf@enzemed.com )}

Taizhou Hospital of Zhejiang Province

\section{Research}

Keywords: SARS-CoV-2, COVID-19, SAA, Monitor 
Posted Date: March 30th, 2020

DOl: https://doi.org/10.21203/rs.3.rs-19724/v1

License: (c) (i) This work is licensed under a Creative Commons Attribution 4.0 International License. Read Full License 


\title{
Serum Amyloid A Protein as a Potential Biomarker Useful in Monitoring the Course of COVID-19: A Retrospectively Studied
}

\author{
Ying Zhang ${ }^{1 *}$, Donglian Wang ${ }^{1 *}$, Minjie Lin ${ }^{1 *}$, Tong Sun, Jiaxi Chen, Jiaqin Xu, Hongguo \\ Zhu, Guangjun Zhu, Ruyue Lu, Luxiao Hong, Bo Shen, Xiaomai Wu ${ }^{1 * *}$, Yufen Zheng ${ }^{1 * *}$
}

1. Taizhou Hospital, Wenzhou Medical University, Linhai, 317000, China
${ }^{* *}$ Correspondence authors: Yufen Zheng, Taizhou Hospital, Wenzhou Medical University, Linhai, 317000, China. Tel+86576 85120120, Email zhengyf@enzemed.com
*These authors contributed equally to this work.

\begin{abstract}
Background: Serum Amyloid A (SAA) is an acute-phase reactant downstream of the proinflammatory cytokines released during virus infection. However, the role of this inflammatory marker in SARA-CoV-2 infection is yet to be elucidated. Here, we explored the potential use of SAA in serum as a biomarker for monitoring the clinical course of COVID-19 patients.

Methods: The subjects included 95 COVID-19 patients discharged from the hospital with acute and / or convalescent phases data, among them 69 patients had paired data. Mann-Whitney U statistics and Wilcoxon signed-rank test were used to compare SAA level in the acute and convalescent phases. A subgroup of COVID-19 patients $(n=9)$ participated in a follow-up examination with repeated blood collection reach five times during the hospitalization. The correlations of SAA levels with laboratory testing were then analyzed using the Spearman test.
\end{abstract}

Results: The results of the data analysis show that the media SAA levels at acute phases were significantly higher $(\mathrm{P}<0.05)$ compared to that at baseline. Furthermore, ascensional range of SAA were associated with the degree of COVID-19 severity. Media SAA levels at convalescent phases were significantly decreased $(\mathrm{P}<0.05)$ compared to that at acute phases. The same phenomenon was seen in patients with and without comorbidities and with fever patients except without fever patients. Furthermore, The SAA concentration change in 9 COVID-19 patients of longitudinal follow-up along with the CT score and SARS-CoV-2 nucleic acid change. In the course of the disease, SAA changes were greater than CRP, lymphocytes, and neutrophils.

Conclusions: The serum SAA levels were found to be significantly correlated with course of the COVID-19, and may serve as a useful biomarker to monitor the complicated clinical course of the disease.

Key words: SARS-CoV-2 COVID-19 SAA Monitor 


\section{Background}

In December 2019, an outbreak of severe respiratory disease was reported in Wuhan, Hubei province of China, and the cause was a new coronavirus, referred to as SARS-CoV-2 [1]. This virus has spread nationally and to other countries as well as raised world concern because of its high transmissibility [2].

The typical symptoms of human infected with SARS-CoV-2 are fever, dry cough, dyspnea, myalgia, fatigue, headache, and pneumonia. In severe cases, the disease can rapidly progress into acute respiratory distress syndrome, septic shock, and metabolic acidosis, coagulation dysfunction, and even death [3]. Reverse transcription-PCR (RT-PCR) of respiratory or stool samples is the most commonly used laboratory diagnostic test for SARS-CoV-2 infection [4], as well as subsequently serological studies of anti-SARS-CoV-2 antibodies clinically applied [5], but they do not seem to play a role in monitoring the course of disease. Although the current series of chest radiographs are helpful in monitoring disease course [6], more objective and simple markers have not been well reported.

SAA is primarily produced by hepatocytes in response to the inflammatory cytokines tumor necrosis factor- $\alpha$ (TNF- $\alpha$ ), interleukin (IL)- $1 \beta$ and IL-6 [7]. In healthy individuals, plasma SAA level is within $0-10 \mathrm{mg} / \mathrm{L}$. However, under inflammatory conditions, plasma SAA levels can increase exponentially, even reaching $1000 \mathrm{mg} / \mathrm{L}$ or more in some cases[8]. The upregulation of SAA expression has been demonstrated in several animal models of viral infection[9]. Similarly, human SAA expression is upregulated during the acute phase of various viral infections, including cytomegalovirus, herpes simplex virus, measles virus, mumps virus, rubella virus and varicella-zoster virus, and returns to normal during the convalescent phase of infection[10]. Pertaining to acute infection, enhanced SAA expression might serve as a mechanism by which the innate immune system attempts to control the infection.

Changes in disease status during the treatment of COVID-19 are closely related to measures taken, which means that it is important to conduct appropriate monitoring of patients after diagnosis of COVID-19, while objective, simple and economical monitoring indicators are still unknown. In this study, we analyzed the serum SAA in COVID-19 patients to subsequently investigate the possible role in disease monitoring.

\section{Methods}

\section{Study population and characteristics}

We performed this retrospectively study in Taizhou Public Health Medical Center (TPHMC), Taizhou Hospital, Zhejiang province, China, which was the designated hospital of Taizhou city. Between January 17, 2020 and February 27, 2020, 144 patients who fulfilled the Diagnostic 
Criteria (trial version 5) for confirmed SARS-COV-2 infection were admitted to the TPHMC. In 99 of these patients (69\%) met discharge standards and Successful discharged. Two patients (2\%) were excluded from this study due to a concurrent acute illness on presentation and two patients $(2 \%)$ were absence of a recorded outcome. Consequently, the final study cohort comprised 95 patients whose serum SAA levels were obtained on admission and discharge, and whose sole acute illness was a confirmed diagnosis of SARS-COV-2 infection (Figure 1).

COVID-19 patients' mean age was $45.1 \pm 14.1$ years. There were $56(59 \%)$ male and 39(41\%) female patients. The most common symptoms on presentation were fever $(n=63,66 \%)$, couch $(\mathrm{n}=51,54 \%)$, and fatigue $(\mathrm{n}=18,19 \%)$. The median time from symptom onset until presentation to the TPHMC was 5 days (IQR 3-8). The median length of hospitalization was 15 days (IQR 10-21) (Table 1). Ninety-two (97\%) patients were with abnormal chest radiograph and fifty (53\%) patients had coexisting conditions. Older age and hospitalization days were linked with severe disease (Table 2; $P=0.06, P=0.000$ ).

\section{Study Design}

To simplify data analysis, the patients were only classified as either general or severe clinical symptom groups based on the guideline newly released by Chinese government. General disease $(n=79)$ included patients with symptoms of fever, cough, and headache, and with radiological evidence of pneumonia. Severe disease $(n=16)$ comprised patients who met any of the following: 1. Respiratory distress, $R R \geq 30 / \mathrm{min} ; 2$. In resting state, oxygen saturation $\leq 93 \%$; 3. Partial arterial oxygen pressure $\left(\mathrm{PaO}_{2}\right)$ /oxygen absorption concentration $\left(\mathrm{FiO}_{2}\right) \leq 300 \mathrm{mmHg}$. In this study, the acute phase was defined as the period of 24 hours after presentation; the convalescent phase was defined as the period of 48 hours before discharge.

Chest CT evaluation: the major CT demonstrations were described using internationally standard nomenclature defined by the Fleischner Society glossary and peer-reviewed literature on viral pneumonia, using terms including ground glass opacity (GGO), crazy-paving pattern, and consolidation [11, 12]. A semi-quantitative scoring system was used to quantitatively estimate the pulmonary involvement of all these abnormalities on the basis of the area involved [13].

\section{Statistical Analysis}

Statistical analysis was performed using Prism version 7.0 software (GraphPad, San Diego, California). Values are presented as means $\pm \mathrm{SD}$ or median and interquartile range (IQR) for the continuous variables and as number of individuals (and the percentage in each group) for 
the categorical variables. The comparison of continuous variables between groups was by independent samples Student's t-test for normally distributed variables and by the MannWhitney U statistics for non-normally distributed variables. The Wilcoxon signed-rank test were used to compare the paired samples. Correlation analysis was carried out using the Spearman test. Differences were considered statistically significant at $P<0.05$.

\section{Results}

\section{Serum SAA concentrations in the acute and convalescent Phases}

The serum concentration of SAA was significantly elevated at the acute phase of infection (8.9-, 4.8-, and 48-fold increase in all patients, general illness and severe illness, respectively), the extent of which was correlated with disease severity, and significantly declined at the convalescent phase though did not reach to basal levels ( in all patients, general illness and severe illness, $P=0.000$ ) (Figure 2a, Table 2). In addition, the increased SAA was observed in patients with severe, especially at the acute phase of infection (median and interquartile range (IQR) of SAA in severe vs general at acute and Convalescent phase: 480.6(118.5-1064.8) vs 47.9(14.1-167.2) and 33.2(12.6-113.7) vs $11.3(7.1-21.8), P=0.000$ and $P=0.003$, respectively). Similarly, the same phenomenon that the serum concentration of SAA was significantly elevated at the acute phase of infection and significantly declined at the convalescent phase was seen in patients with and without comorbidities and with fever patients except without fever patients (Table 2). The increased SAA was observed in patients with fever, especially at the acute phase of infection (120.5(31.7-246.6) vs $14.8(7.4-117.9)$ and $15.8(7.9-42.7)$ vs 9.1(6.717.9), $P=0.000$ and $P=0.047$ in acute and Convalescent phase respectively). The same laboratory observation was noted in regard to patients who had coexisting conditions compared to those who did not; however, in this comparison, the difference was not statistically significant.

\section{Serum SAA concentrations in the acute and convalescent phases of matched patients}

We also analyzed serum SAA concentrations in 69 matched patients in the acute and convalescent phases. As shown in Figure 3, SAA expression was significantly different in the acute phase and the convalescent phase in the same patient, whether in all patients or in a variety of grouping.

\section{Dynamic changes of Serum SAA concentrations and CT score in the course of disease}

After admission, 9 patients were continuously monitored for five times (within 24 hours of 
admission, treatment course 1 , treatment course 2 , after the negative conversion of real-time RT-PCR and within 48 hours of discharge), we correlated its concentrations with the clinical findings and serial chest radiographic scores in 9 COVID-19 patients.

After SARS-CoV-2 infection was diagnosed, the radiographic score median of these patients under longitudinal follow-up started to increase from a value of 9.3 to a peak of 11.2 and then gradually decreased to $10.1,8.3$, and finally to 5.8, demonstrating a progressive recovery (Fig. 4). The SAA concentration showed an increase that peaked later than the radiographic score but then also gradually decreased along with the radiographic score to a nadir when the patient was discharged.

\section{Dynamic changes of Serum SAA concentrations and laboratory testing in the course of disease}

Among the laboratory markers, CRP, neutrophils, lymphocytes, and monocytes were followed. As shown in figure 5, SAA has a significant trend of change, reaching a peak in treatment process 3 and then rapidly decreasing. CRP slowly peaks at treatment 2 and then slowly declines. Neutrophils have a similar trend, but they peak at treatment 3 and change more slowly. Because the range of change is very small that lymphocytes can hardly see the trend, and monocytes have no regular trend at all.

In the course of disease, the concentration of SAA decreased from the peak in treatment process 3 and to a nadir in line with SARS-Cov-2 nucleic acid changed from positive to negative in treatment process 3 (Fig. 5).

\section{Serum SAA association with the laboratory testing in COVID-19 patients}

We analyzed the correlation between SAA levels and laboratory tests in the acute and convalescent phases. As shown in figure 6, CRP and lymphocytes were significantly correlated with SAA in both the acute phase and the convalescent phases $(P<0.01)$. Neutrophils were significantly associated with SAA only in the acute phase $(P=0.01)$. However, there was no significant correlation between mononuclear cells and SAA in both the acute phase and the convalescent phases.

\section{Discussion}

Changes in disease status during treatment in COVID-19 mean that it is important that patients are appropriately monitored after a diagnosis of COVID-19; therefore, the discovery of acutephase proteins that are strongly associated with inflammatory events is expected and entirely matches with the theme of this study in disease monitoring. Serial chest radiographs are helpful 
in the diagnosis and monitoring of the clinical course [6], but these explanations can be confusing because of the lack of experienced radiologists, the differences between observers, and the often sub-optimal quality of portable film taken in isolation wards to prevent the infection from spreading to other patients. SARS-CoV-2 antibody is usually increased at a later stage of disease [5] and therefore cannot be a good marker for monitoring disease levels. Because high titers predict the need for intensive care, it has been suggested that quantitative real-time RT-PCR to determine the viral load may have prognostic value $[14,15]$. However, because the damage of SARS-CoV-2 appears to be more related to the immunopathology of the host reaction than to the replication of the virus, its application in disease surveillance may be limited or at least unclear.

As an acute-phase protein synthesized by the liver, SAA has been reported to be increased in infectious and arthritic diseases and malignancies [16]. Our observations confirmed a significant increase in serum SAA levels during the acute phase, thus strengthening the association. Thus, the elevated serum levels of SAA, which are associated with inflammatory process and recruitment of inflammatory cells, could reflect the ongoing inflammation at the infection site. Pulmonary fibrosis were found in the COVID-19 patients [17]. It is also widely known that such damage can serve as stimuli for the production and secretion of a variety of inflammatory cytokines. COVID-19 patients have been shown to have markedly increased plasma concentrations of two inflammatory cytokines, interleukin-6 (IL-6) [3]. Knowing that IL-6 can rapidly induce 1000-fold increases in SAA in a synergistic manner, it thus perhaps explains the possible rapid induction of this acute-phase reactant at the time of SARS-CoV-2 infection.

Additionally, the elevation of SAA was associated with disease severity and fever. Interestingly, the plasma level of SAA was detected to be no difference between with and without Comorbidities irrespective of disease state. It is possible that underlying diseases have little effect on the inflammatory response induced by sras-cov- 2 .

As the treatment progressed, the condition gradually improved and SAA levels significantly decreased at the convalescent phase. However, when discharged from the hospital, SAA levels did not decline to normal levels. We speculate that although the condition has recovered to the discharge standard, the inflammatory response of the body continues. The precise mechanism for the changes of SAA concentrations in COVID-19 patients remains to be investigated and further studies will be needed to address these possibilities.

Using clinically useful chest radiograph scores, we found a good correlation between SAA concentration and the degree of pneumonia in 9 patients. However, the inflection point of its 
decline was later than the $\mathrm{CT}$ score, this is contrary to the change of SAA concentration in SARS patients earlier than the CT score reported by Timothy T.C. Yip. We speculate that this late decrease of SAA might be associated with the systemic inflammation, not confined to the lungs, although this remains to be confirmed in subsequent studies.

Apart from SAA, other candidate acute-phase proteins, such as C-reactive protein, exist in various mammals[18]. SAA was found to be more sensitive than C-reactive protein in detecting minor inflammatory stimuli in certain viral infections [19] and in noninvasive and early invasive bacterial infections[20]. A study of feline disease conditions also showed that SAA was the earliest marker induced when compared with C-reactive protein[18].

SAA and other biomarkers that may be discussed can be used to monitor the course of disease and response to treatment in COVID-19 patients as the disease progresses and as the remaining serum biomarkers increase or decrease in relation to the possible association with the disease state. Our study had limiting factors. The design of this study was retrospective study, which did not allow us to accurately determine when seroconversion occurred or when viral RNA ceased to be detectable.

\section{Conclusions}

In the current sample of patients with pandemic SARS-Cov-2 infection, the serum SAA levels obtained at admission and discharge were found to be significantly correlated with impending course of the disease. Based on this finding, it is plausible that this measurement may serve as a useful biomarker to monitor a complicated clinical course of the disease.

\section{Abbreviations}

SAA: Serum Amyloid A; TPHMC: Taizhou Public Health Medical Center; CRP: C reactive protein.

\section{Declarations}

\section{Ethics approval and consent to participate}

The present study was approved by the ethics review committee of Taizhou hospital in Zhejiang Province, and informed consent was obtained from each subject

\section{Consent for publication}

The present study had consent for publication.

\section{Availability of data and materials}


The datasets used and/or analysed during the current study are available from the corresponding author on reasonable request.

\section{Competing interests}

The authors declare that they have no competing interests.

\section{Funding}

This work was supported by grants from the National Natural Science Foundation of China (81672086) and Zhejiang Province Analysis Test Project (2018C37032).

\section{Authors' contributions}

ZY participated in the conception, design, analysis of data and manuscript drafting; WDL participated in acquisition of data and conception of the study; LMJ carried out the ranking and interpretation of chest radiographs; ST and ZGJ participate in laboratory testing; CJX performed the statistical analysis and participated in the interpretation of data and manuscript drafting; XJQ, ZHG, LRY participated in the coordination of the study; HLX and WXM participate in clinical working; SB coordinated study and revised the manuscript for important intellectual content; ZYF took part in the study design and revised the manuscript for important intellectual content. All authors read and approved the final manuscript.

\section{Acknowledgements}

Not applicable. 


\section{References}

1 Zhu N, Zhang D, Wang W, Li X, Yang B, Song J, Zhao X, Huang B, Shi W, Lu R, Niu P, Zhan F, Ma X, Wang D, Xu W, Wu G, Gao GF, Tan W, China Novel Coronavirus I, Research T. A Novel Coronavirus from Patients with Pneumonia in China, 2019. N Engl J Med. 2020; 382:727-733.

2 Giovanetti M, Benvenuto D, Angeletti S, Ciccozzi M. The first two cases of 2019-nCoV in Italy: Where they come from? J Med Virol. 2020.

3 Huang C, Wang Y, Li X, Ren L, Zhao J, Hu Y, Zhang L, Fan G, Xu J, Gu X, Cheng Z, Yu T, Xia J, Wei Y, Wu W, Xie X, Yin W, Li H, Liu M, Xiao Y, Gao H, Guo L, Xie J, Wang G, Jiang R, Gao Z, Jin Q, Wang J, Cao B. Clinical features of patients infected with 2019 novel coronavirus in Wuhan, China. The Lancet. 2020; 395:497-506.

4 Paulini I, Siqueira-Silva J, Thomaz L, Rocha L, Harsi C, Bellei N, Granato C. Development of a prototype immunochromatographic test for rapid diagnosis of respiratory adenovirus infection. The Brazilian Journal of Infectious Diseases. 2017; 21:500-506.

5 Li Z, Yi Y, Luo X, Xiong N, Liu Y, Li S, Sun R, Wang Y, Hu B, Chen W, Zhang Y, Wang J, Huang B, Lin Y, Yang J, Cai W, Wang X, Cheng J, Chen Z, Sun K, Pan W, Zhan Z, Chen L, Ye F. Development and Clinical Application of A Rapid IgM-IgG Combined Antibody Test for SARS-CoV-2 Infection Diagnosis. J Med Virol. 2020.

6 Pan F, Ye T, Sun P, Gui S, Liang B, Li L, Zheng D, Wang J, Hesketh RL, Yang L, Zheng C. Time Course of Lung Changes On Chest CT During Recovery From 2019 Novel Coronavirus (COVID-19) Pneumonia. Radiology. 2020:200370.

7 Jensen LE, Whitehead AS. Regulation of serum amyloid A protein expression during the acute-phase response. Biochem J. 1998; 334 ( Pt 3):489-503.

8 De Buck M, Gouwy M, Wang JM, Van Snick J, Opdenakker G, Struyf S, Van Damme J. Structure and Expression of Different Serum Amyloid A (SAA) Variants and their Concentration-Dependent Functions During Host Insults. Curr Med Chem. 2016; 23:1725-1755.

9 Pomorska-Mol M, Markowska-Daniel I, Kwit K, Stepniewska K, Pejsak Z. C-reactive protein, haptoglobin, serum amyloid A and pig major acute phase protein response in pigs simultaneously infected with H1N1 swine influenza virus and Pasteurella multocida. BMC Vet Res. 2013; 9:14.

10 Sarov I, Shainkin-Kestenbaum R, Zimlichman S, Winikoff Y, Chaimovitz C, Pras M. Serum amyloid A levels in patients with infections due to cytomegalovirus, varicella-zoster virus, and herpes simplex virus. J Infect Dis. 1982; 146:443.

11 Franquet T. Imaging of pulmonary viral pneumonia. Radiology. 2011; 260:18-39.

12 Hansell DM, Bankier AA, MacMahon H, McLoud TC, Muller NL, Remy J. Fleischner Society: glossary of terms for thoracic imaging. Radiology. 2008; 246:697-722.

13 Chang YC, Yu CJ, Chang SC, Galvin JR, Liu HM, Hsiao CH, Kuo PH, Chen KY, Franks TJ, Huang KM, Yang PC. Pulmonary sequelae in convalescent patients after severe acute respiratory syndrome: evaluation with thin-section CT. Radiology. 2005; 236:1067-1075.

14 Corman VM, Landt O, Kaiser M, Molenkamp R, Meijer A, Chu DK, Bleicker T, Brunink S, Schneider J, Schmidt ML, Mulders DG, Haagmans BL, van der Veer B, van den Brink S, Wijsman L, Goderski G, Romette JL, Ellis J, Zambon M, Peiris M, Goossens H, Reusken C, Koopmans MP, Drosten C. Detection of 2019 novel coronavirus (2019-nCoV) 
by real-time RT-PCR. Euro Surveill. 2020; 25.

15 Chu DKW, Pan Y, Cheng SMS, Hui KPY, Krishnan P, Liu Y, Ng DYM, Wan CKC, Yang P, Wang Q, Peiris M, Poon LLM. Molecular Diagnosis of a Novel Coronavirus (2019-nCoV) Causing an Outbreak of Pneumonia. Clin Chem. 2020. 16 Zhang Y, Zhang J, Sheng H, Li H, Wang R. Acute phase reactant serum amyloid A in inflammation and other diseases. Adv Clin Chem. 2019; 90:25-80.

17 Wang J, Wang BJ, Yang JC, Wang MY, Chen C, Luo GX, He WF. [Advances in the research of mechanism of pulmonary fibrosis induced by Corona Virus Disease 2019 and the corresponding therapeutic measures]. Zhonghua Shao Shang Za Zhi. 2020; 36:E006.

18 Kajikawa T, Furuta A, Onishi T, Tajima T, Sugii S. Changes in concentrations of serum amyloid A protein, alpha 1 -acid glycoprotein, haptoglobin, and C-reactive protein in feline sera due to induced inflammation and surgery. Vet Immunol Immunopathol. 1999; 68:91-98.

19 Nakayama T, Sonoda S, Urano T, Yamada T, Okada M. Monitoring both serum amyloid protein A and C-reactive protein as inflammatory markers in infectious diseases. Clin Chem. 1993; 39:293-297.

20 Lannergard A, Larsson A, Kragsbjerg P, Friman G. Correlations between serum amyloid A protein and C-reactive protein in infectious diseases. Scand J Clin Lab Invest. 2003; 63:267-272.

\section{Figures, tables}

Fig 1 Study flowchart

Figure 2: Serum SAA concentrations in the acute and convalescent phases

Serum SAA concentrations of 95 COVID-19 patients in the acute and convalescent phases, (a) in all patients, general disease and sever disease; (b) in patients with fever and without fever; (c) in patients with and without comorbidities.

Figure 3: Serum SAA concentrations in the acute and convalescent phases of matched patients

Serum SAA concentrations of 95 COVID-19 patients in the acute and convalescent phases of matched patients, (a) in all patients, general disease and sever disease; (b) in patients with fever and without fever; (c) in patients with and without comorbidities.

Figure 4: Dynamic changes of Serum SAA concentrations and CT score in the course of disease

Course of COVID-19, (a) SAA level and CT score of nine COVID-19 patients; (b) a typical continuous CT image of a patient. Sputum/Throat Swab CoV +ve or -ve: RT PCR positive or negative for SARS-CoV-2 in sputum/throat swab. Course of disease, 1: Admission; 2: Treatment course 1; 3: Treatment course 2; 4: After the negative conversion of realtime RT-PCR; 5: Discharge.

Figure 5: Dynamic changes of laboratory testing in the course of disease

SAA level and laboratory testing of nine COVID-19 patients in the course of COVID-19.

Sputum/Throat Swab CoV +ve or -ve: RT PCR positive or negative for SARS-CoV-2 in sputum/throat swab. Course of disease, 1: Admission; 2: Treatment course 1; 3: Treatment course 2; 4: After the negative conversion of real-time RT-PCR; 5: Discharge.

Figure 6: Serum SAA association with the laboratory testing in COVID-19 patients 
Figures

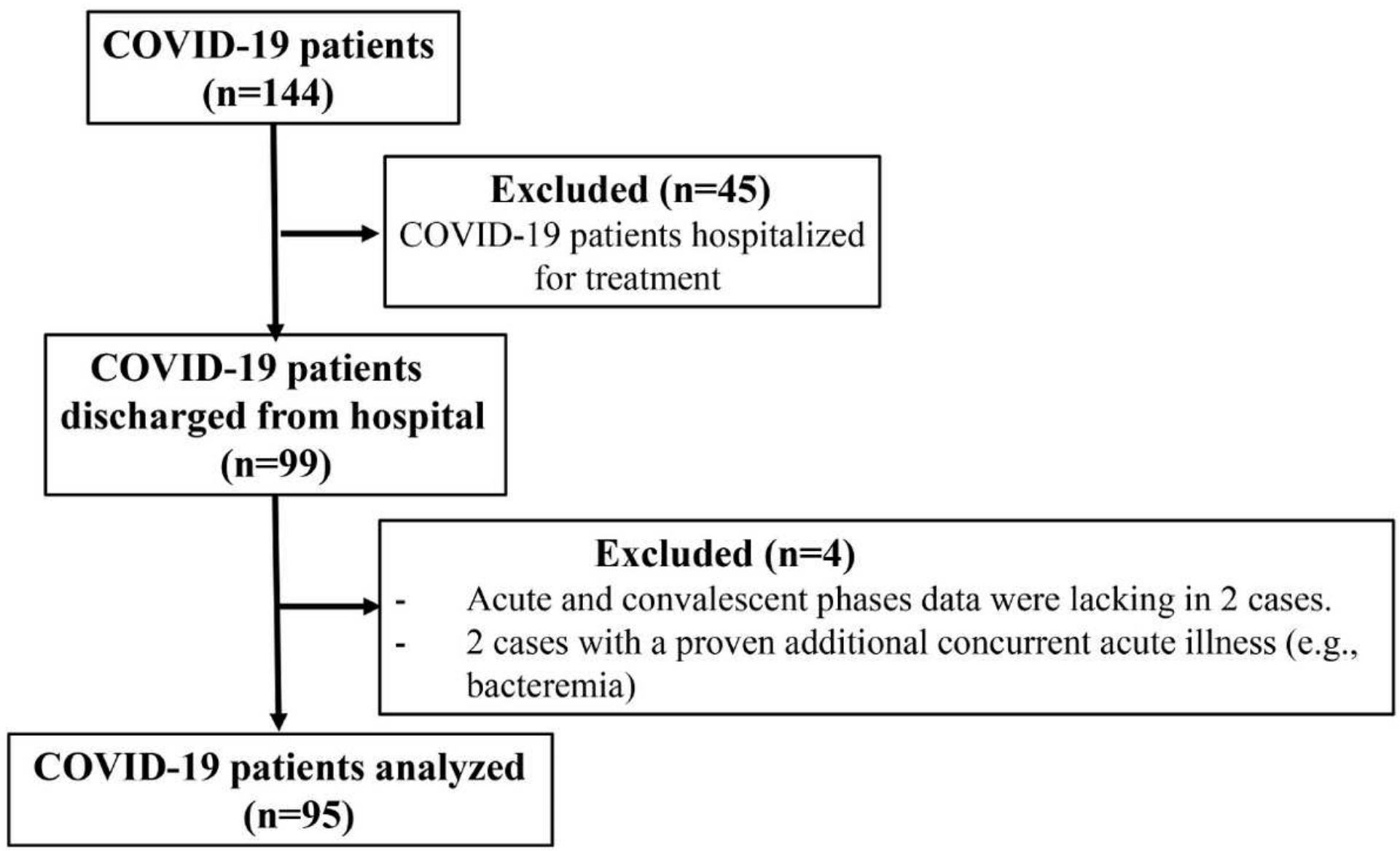

Figure 1

Study flowchart 
a.

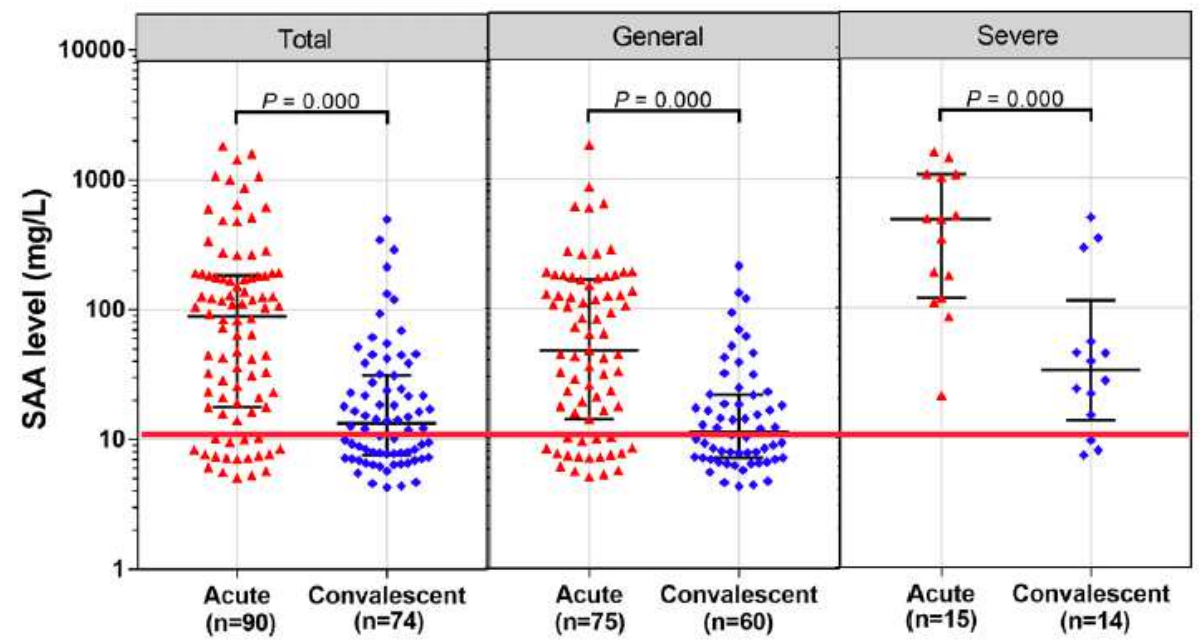

b.
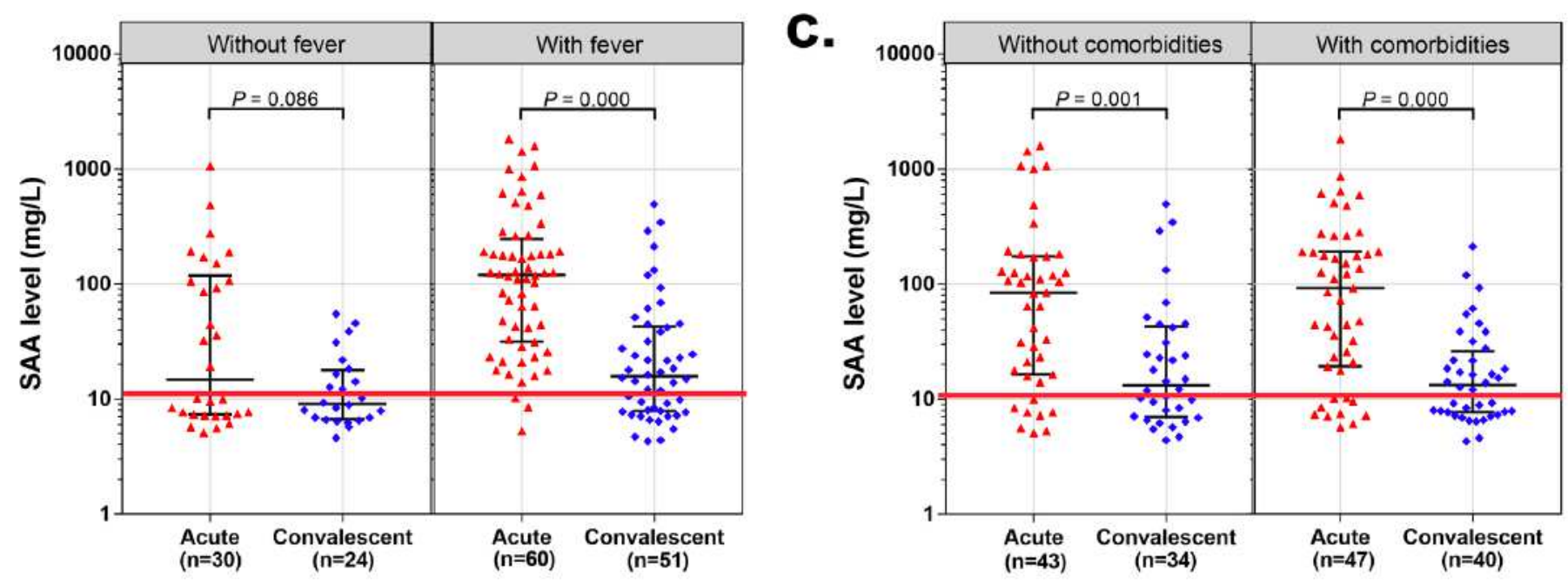

Figure 2

Serum SAA concentrations in the acute and convalescent phases Serum SAA concentrations of 95 COVID-19 patients in the acute and convalescent phases, (a) in all patients, general disease and sever disease; (b) in patients with fever and without fever; (c) in patients with and without comorbidities. 
a.
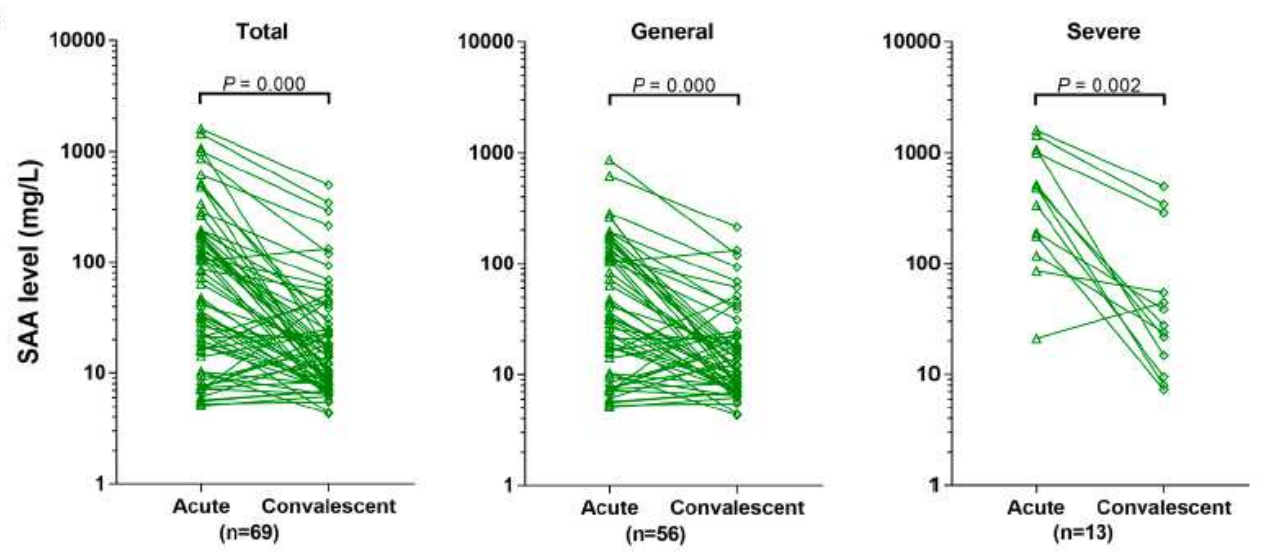

b.
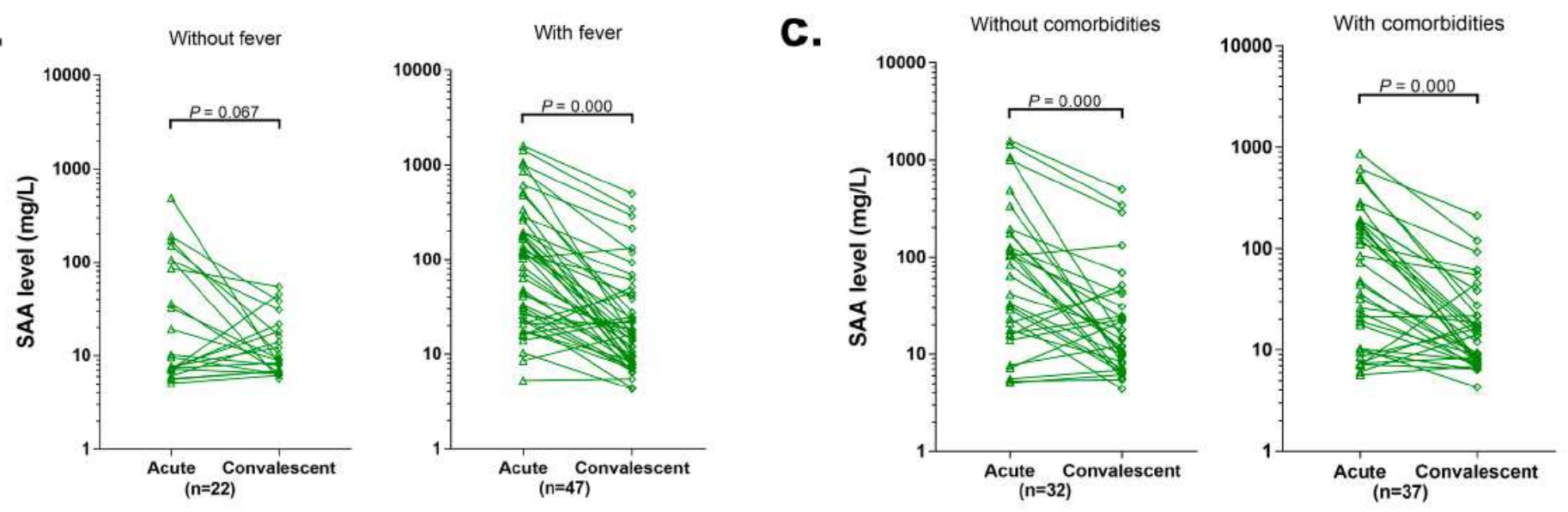

Figure 3

Serum SAA concentrations in the acute and convalescent phases of matched patients Serum SAA concentrations of 95 COVID-19 patients in the acute and convalescent phases of matched patients, (a) in all patients, general disease and sever disease; (b) in patients with fever and without fever; (c) in patients with and without comorbidities. 
a.

Course of disease:
Sputum/Throat Swab $\mathrm{CoV}+\mathrm{ve}$
Sputum/Throat Swab

CoV -ve

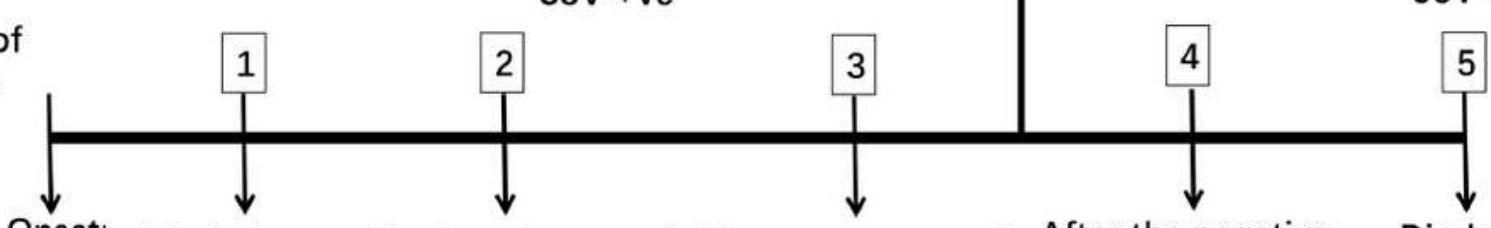

Onset: Admission Treatment course 1 Treatment course 2 After the negative Discharge

Fever cough

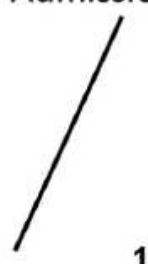
conversion of real-time RT-PCR

SAA level and CT score of nine COVID-19 patients

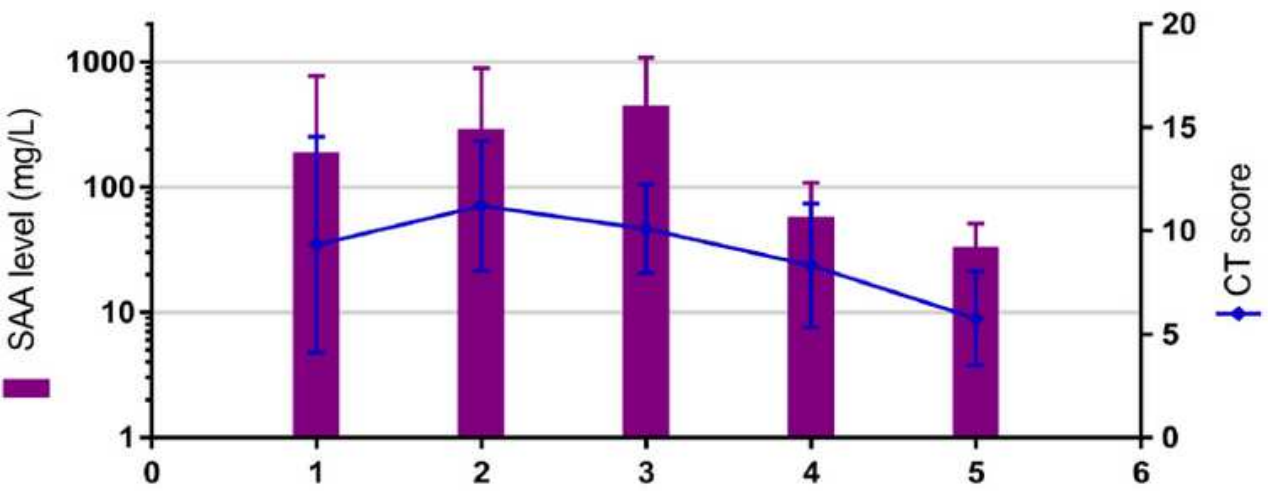

Course of disease

b.

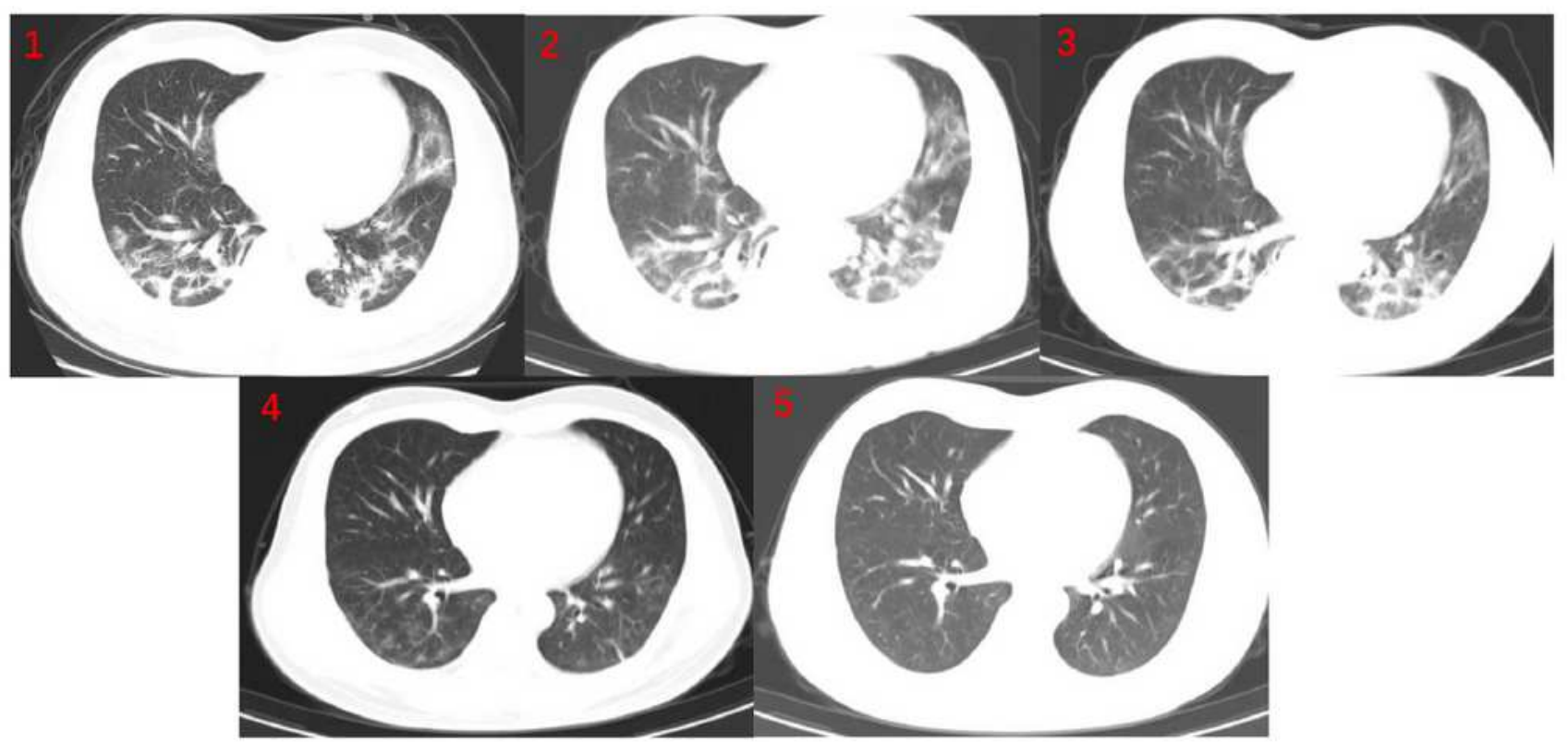

Figure 4

Dynamic changes of Serum SAA concentrations and CT score in the course of disease Course of COVID19, (a) SAA level and CT score of nine COVID-19 patients; (b) a typical continuous CT image of a patient. Sputum/Throat Swab CoV +ve or -ve: RT PCR positive or negative for SARS-CoV-2 in sputum/throat swab. Course of disease, 1: Admission; 2: Treatment course 1; 3: Treatment course 2; 4: After the negative conversion of real-time RT-PCR; 5: Discharge. 


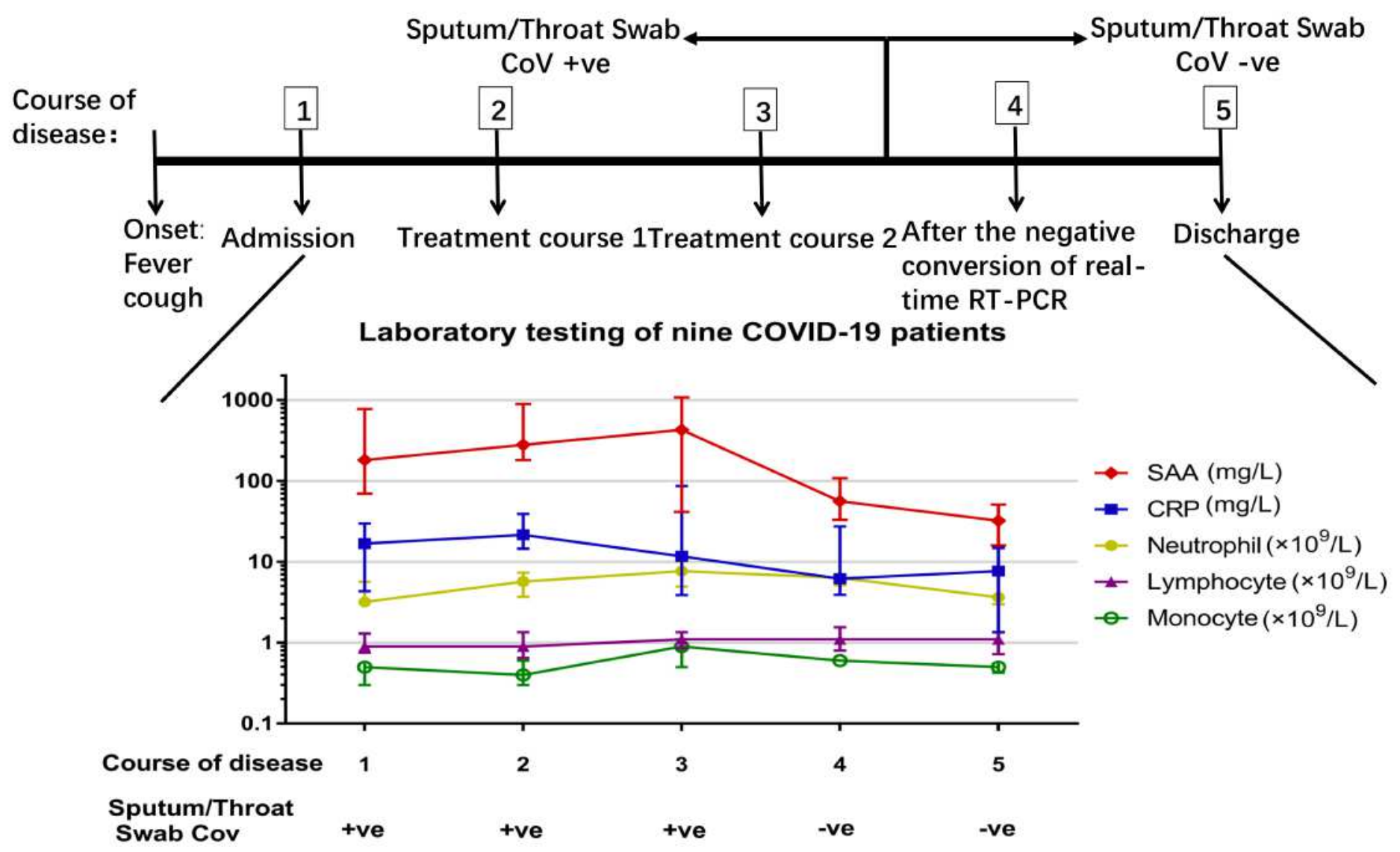

\section{Figure 5}

Dynamic changes of laboratory testing in the course of disease SAA level and laboratory testing of nine COVID-19 patients in the course of COVID-19. Sputum/Throat Swab CoV +ve or -ve: RT PCR positive or negative for SARS-CoV-2 in sputum/throat swab. Course of disease, 1: Admission; 2: Treatment course 1; 3: Treatment course 2; 4: After the negative conversion of real-time RT-PCR; 5: Discharge. 
In the acute phases:

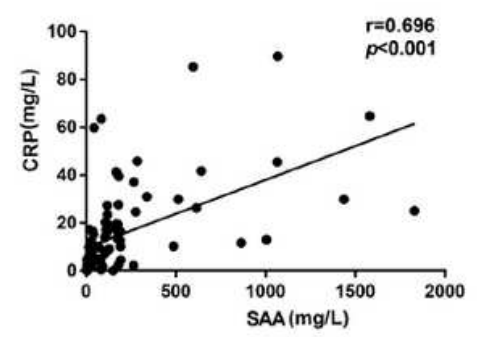

In the convalescent phases:
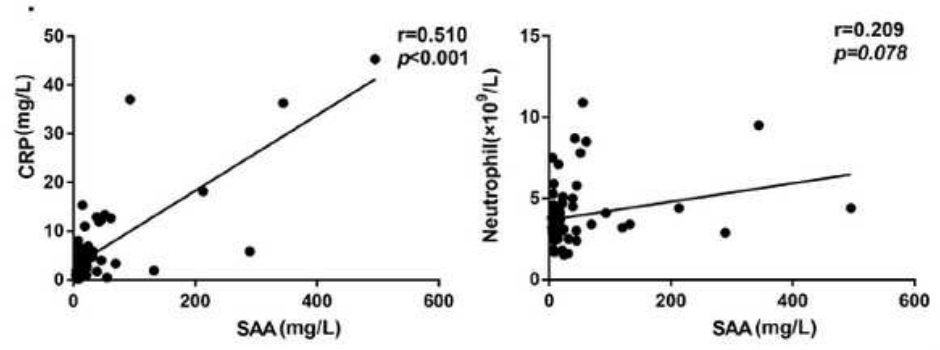

(b)
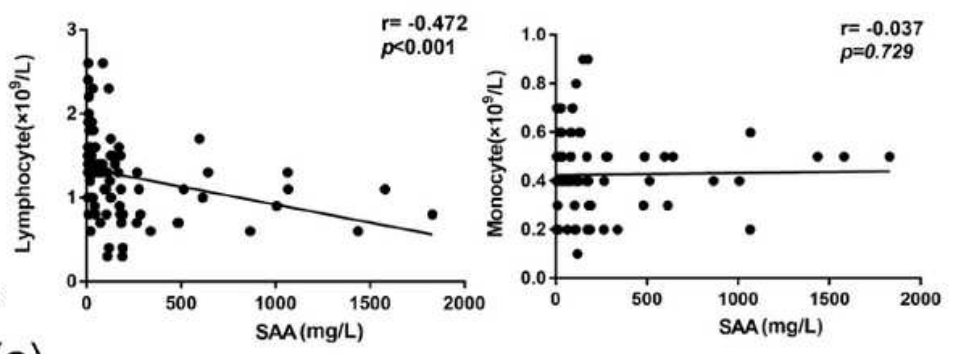

(a)
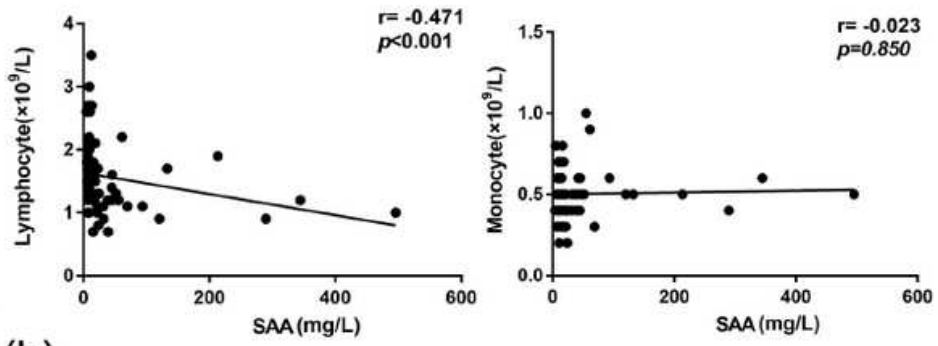

Figure 6

Serum SAA association with the laboratory testing in COVID-19 patients 